2. "Hirschartige und Landeskultur",

Obmann: Dr. J. A. Eygenraam, I.T.B.O.N., Kamperbergweg 11, Arnheim, Holland.

3. „Die jagdliche Ausbeutung der Zugvögel",

Obmann: Dr. G. V. T. Matrinews, The Wildfowl Trust, Slimbridge,

Gloucestershire, England.

Da für jedes Thema nur eine beschränkte Zahl von Vorträgen angenommen werden kann, wird empfohlen, sich bald mit dem betreffenden Obmann in Verbindung zu setzen.

Die übrige Zeit des Kongresses steht für Vortragsthemen nach eigener Wahl zur Verfügung, die zu gegebener Zeit bei dem Verbindungsmann für das Vereinigte Königreich, Charles L. Coles, Kynoch Game Advisory Service, Fordingbridge, Hampshire, England, anzumelden sind.

Eine weitere und endgültige Mitteilung über Zeitpunkt und Dauer des Kongresses, über Exkursionen und sonstige Einzelheiten wird an dieser Stelle erscheinen, wenn genaue Angaben feststehen.

\title{
Dr. W. Rieck zum Professor ernannt
}

Der Niedersächsische Kultusminister hat den Dozenten für Jagdkunde an der Universität Göttingen, Dr. W. RrEck, zum außerplanmäßigen Professor ernannt.

Prof. RIEcK ist Mitarbeiter beim Institut für Jagdkunde der Forstlichen Fakultät der Universität Göttingen in Hann. Münden.

\section{REFERATE}

Referenten: v. Braunschweig (v.B.), BrunX (Bk.), Lindner (Ld.), Nüsslein (Nn.), Rieck (Rk.)

\section{O. Jagdkunde}

Gagern, F. y.: Das Jahrbuch der Jäger. Verlag Das Bergland-Buch, Salzburg/Stuttgart, 1962. 326 S. 19,80 DM.

Das Buch enthält eine bunte Zusammenstellung von Berichten, in denen z. T. spezielle Angaben, z. B. die Jahresstrecken, für das Jagdjahr 1960/61 enthalten sind. Über Wild und Jagd folgender Gebiete wird eine Ubersicht gegeben: Schleswig-Holstein, Niedersachsen, NordrheinWestfalen, Hessen, Bayern, Osterreich und Ungarn. Weitere Kapitel beschäftigen sich mit dem Wild der Gegenwart in Tirol, dem Rotwild in Slowenien und Slawonien sowie der Jagd und dem Rotwild in Rumänien. Besondere Ausführungen werden über die Geschichte des Wildparks bei Erbach (Odenwald), das Schälen des Rotwildes im Spiegel der Forschung, die zulässige Rotwilddichte und über den Begriff der Waidgerechtigkeit gebracht.

Die Darstellungen über Wild und Jagd der einzelnen Länder sind unterschiedlich, häufig steht das Rotwild im Vordergrund der Betrachtungen, das aus zahlreichen Rotwildringen und Vorkommensgebieten geschildert wird. An Besonderheiten bringt Schleswig-Holstein eine Darstellung der gegenwärtigen Landschaft sowie Mitteilungen über die Jägerschaft, den Jägerhof und die Forschungsstation. Niedersachsen führt in die Geschichte der Jagd ein und läßt Berichte aus fünf einzelnen Jagdkreisen folgen. Nordrhein-Westfalen bringt ebenfalls Angaben aus der Geschichte von Wild und Jagd, ferner über die Forschungsstelle für Jagdkunde und über die Wilderei. Hessen führt die Jagdschlösser des 15. bis 18. Jahrhunderts auf, berichtet über die Arbeit der Hessischen Jägerschaft und den Wettbewerb im Jagdhornblasen. Bayern bringt Ausführungen über den Jagdschutzgedanken, die Pflichttrophäenschau und den Berufsjäger. Tirol schildert die Zeiten Kaiser Maximilians und bringt aus der Gegenwart Angaben uiber das Steinwild, über Wilderer und über Größen und Besitzverhältnisse der Jagdflur. Aus Ungarn und 